\title{
Optics-based compressibility parameter for pharmaceutical tablets obtained with the aid of the terahertz refractive index
}

Mousumi Chakraborty ${ }^{1 *}$, Cathy Ridgway ${ }^{2}$, Prince Bawuah ${ }^{1}$, Daniel Markl ${ }^{3}$, Patrick A.C. Gane $^{2,4}$, Jarkko Ketolainen ${ }^{5}$, J. Axel Zeitler ${ }^{3}$, Kai-Erik Peiponen ${ }^{1}$

${ }^{1}$ Institute of Photonics, University of Eastern Finland, P.O. Box 111, FI-80101 Joensuu, Finland.

${ }^{2}$ Omya International AG, CH-4665 Oftringen, Switzerland.

${ }^{3}$ Department of Chemical Engineering and Biotechnology, University of Cambridge, Cambridge CB3 0AS, United Kingdom.

${ }^{4}$ Aalto University, School of Chemical Engineering, Department of Bioproducts and Biosystems, FI-00076 Aalto, Helsinki, Finland.

${ }^{5}$ School of Pharmacy, Promis Centre, University of Eastern Finland, P. O. Box 1617, FI-70211, Kuopio, Finland.

\begin{abstract}
The objective of this study is to propose a novel optical compressibility parameter for porous pharmaceutical tablets. This parameter is defined with the aid of the effective refractive index of a tablet that is obtained from non-destructive and contactless terahertz ( $\mathrm{THz})$ time-delay transmission measurement. The optical compressibility parameter of two training sets of pharmaceutical tablets with a priori known porosity and mass fraction of an active pharmaceutical ingredient (API) was investigated. Both pharmaceutical sets were compressed with microcrystalline cellulose (MCC) and the API Indomethacin. The optical compressibility clearly correlates with the bulk modulus determined by mercury porosimetry and the recently proposed terahertz lumped structural parameter calculated from terahertz measurements. This lumped structural parameter can be used to analyse the pattern of arrangement of excipient and API particles in porous pharmaceutical tablets. Therefore, we propose that the optical compressibility can serve as a quality parameter of a pharmaceutical tablet corresponding with the bulk modulus of the porous tablet, which is related to structural arrangement of the powder particles and the bulk modulus of a porous tablet.
\end{abstract}

\footnotetext{
* Corresponding author: Mousumi Chakraborty

Tel: $+358466379113,+358505270240$,

Email Address: mousumi.chakraborty@uef.fi
} 


\section{Introduction}

For pharmaceutical applications tablets are the accepted and most widely used dosage form due to their being cost effective to manufacture, having relative ease of large scale of production, resulting product stability, related to the availability of reliable manufacture processes, and ability to provide correct reproducible dosage of drug from tablet to tablet and the convenience for patients $[1,2]$. Critical quality attributes, such as disintegration time or amount of API dissolved after a certain time, are linked to their physical, mechanical, chemical, biological and also optical properties. During formation of a tablet, the mixture of drug and excipient particles is compacted, usually directly or following a granulation step, into a stable porous solid.

Historically, mechanical properties have played an important role in order to assess the functionality of a pharmaceutical tablet following the compaction step. Indentation, elasticity, tensile strength, brittle fracture index, bonding index, strain index, viscoelasticity, compressibility, compatibility, and tabletability are among the various mechanical properties of a tablet that have been explored in depth [3 - 8]. The mechanical properties of pharmaceutical tablets can be described by the relationship between the applied force during the compression and the resulting plastic deformation, and inter-particle bonding within the tablet [9]. These dictate the behaviour of pharmaceutical powder mixtures both during and after compaction. Stress and strain are the basic mechanical properties to describe the relationship between compressive pressure and the resulting deformation [8]. Compressibility (solid fraction as a function of compaction pressure) and compactibility (tensile strength in relation to solid fraction) [10] are terms commonly used to describe the densification and reduction in volume of a powder bed by the application of pressure alone, and both properties are considered to be the major parameters contributing to tabletability, defined as the dependence of tensile strength on compaction pressure [11]. In this study, we propose to establish an optical parameter that is related to the mechanical properties, such as the bulk modulus of pharmaceutical tablets. This topic of high importance in pharmaceutical sciences (see, for example, reference to strain [4 $8,10]$ and compressibility [12 - 18]). In this study, the emphasis is on the research of the development of non-contact sensing and data analysis methods to quantify structural and mechanical properties of pharmaceutical tablets using terahertz $(\mathrm{THz})$ time-delay measurement techniques [19].

Recently, we have introduced a novel structural parameter $(S)$, which describes the pattern of arrangement of different constituents in porous pharmaceutical tablets [20]. By pattern arrangement we mean the arrangement of drug and excipient constituting the skeletal-pore elements (solid phase) in series, parallel or a mix of both patterns. This structural parameter is assumed to play an important role both in the compressibility of a tablet, and in the description of the ingress and permeation of liquids in pharmaceutical tablets. In addition to developing the optical compressibility parameter, we consider in more detail the structural parameter $S$ in respect to the explicit dependence of $S$ on a range of various tablet properties, and analyse the correlation of the optical compressibility parameter with $S$. 
This study continues our work to retrieve physical parameters, which directly affect critical quality attributes of a tablet, from non-destructive and contactless terahertz measurements. So far, we have established correlation between the effective $\mathrm{THz}$ refractive index and porosity [21], surface roughness [21], lumped structural parameter [20], and Young's modulus [8]. Here, we suggest a new optical compressibility parameter and compare it with the measured bulk modulus of tablets.

\section{Theory}

The data analysis in this study is based on the measurement of time delay $(\Delta t)$ of a terahertz pulse. The time delay is caused by the more optically dense tablet compared to the undisturbed propagation of the pulse through nitrogen gas, which is typically used as a reference medium in laboratory terahertz measurements. Hence, we assume the validity of the following equation

$$
\left(n_{\mathrm{eff}}-1\right) H=c \Delta t
$$

where $n_{\text {eff }}$ is the effective refractive index of the tablet, $H$ is the height of the round flat-faced tablet, corresponding in direction to the normal of incidence, and $c$ is the velocity of light in vacuum. The refractive index of nitrogen is assumed to be equal to unity.

In the derivation of the structural parameter $S$ of a porous pharmaceutical tablet we exploited the concept of effective permittivity of the tablet and Wiener bounds that define the boundary range for the effective permittivity in the absence of scattering of the terahertz waves. Aspnes [22] provides a nice description of Wiener bounds for composite materials by considering two limiting cases, namely no screening and maximum screening of microstructures in the direction of the external electric field. This means that, for example, a needle-shaped particle orientated parallel to the external electric field (in our case direction of propagation of the $\mathrm{THz}$ pulse) would develop little screening, whereas a disc-shaped particle of the same volume would yield strong screening. The effective permittivity of a porous pharmaceutical tablet can be assumed to be constructed from parallel and series connections of the internal solid structures as follows [20]:

$$
\varepsilon_{\text {eff }}=\frac{1}{\frac{1-\mathrm{S}}{\varepsilon_{\mathrm{U}}}+\frac{\mathrm{S}}{\varepsilon_{\mathrm{L}}}}
$$

where $\varepsilon_{\mathrm{U}}$ and $\varepsilon_{\mathrm{L}}$ are the upper and lower Wiener bounds of the permittivity, respectively, and $S$ is the structural parameter. $S$ is a measure of that fractional part of the randomly distributed structures in a porous medium that can be lumped together in parallel and in series coordination, respectively. Since the true value of the effective permittivity of the tablet is always confined between the upper and lower values of the effective permittivity, the structural parameter $S$ is a number that ranges from zero (all constituents in parallel) to one (all constituents in series). The definition of $S$ holds equally for multiphase systems. In our study, we will only deal with a three-phase system, air and two solid phases, respectively. Eq. (2) was originally defined for effective heat conductivity [23] of porous media, such as coated paper products, but for the sake of analogy we have modified the concept for this analogous case, namely to represent the effective permittivity of porous media. 
In the case of a three-phase system, such as air (or nitrogen gas), micro-crystalline cellulose (MCC) and active pharmaceutical ingredients (API) in this study, the equations for the upper and lower Wiener bounds of the effective refractive index are as follows:

$$
n_{\mathrm{U}}^{2}=f_{\mathrm{air}}+f_{\mathrm{MCC}} n_{\mathrm{MCC}}^{2}+f_{\mathrm{API}} n_{\mathrm{API}}^{2}
$$

and

$$
\frac{1}{n_{\mathrm{L}}^{2}}=f_{\mathrm{air}}+\frac{f_{\mathrm{MCC}}}{n_{\mathrm{MCC}}^{2}}+\frac{f_{\mathrm{API}}}{n_{\mathrm{API}}^{2}}
$$

where $f_{\text {air }}, f_{\mathrm{MCC}}$ and $f_{\mathrm{API}}$ are the volume fractions of air (i.e. the pores constituting the tablet porosity), MCC and API, respectively. The symbols $n_{\mathrm{MCC}}$ and $n_{\mathrm{API}}$ denote the intrinsic refractive indices of MCC and API. If we apply the well-known relation from optics for the real relative permittivity and the refractive index of a non-absorbing insulating medium, namely, $n=\sqrt{\varepsilon}$, we get from Eqs. (2)-(4) the expression

$$
S=\frac{1}{n_{\mathrm{U}}^{2}-n_{\mathrm{L}}^{2}}\left[\frac{n_{\mathrm{U}}^{2} n_{L}^{2}}{n_{\mathrm{eff}}^{2}}-n_{\mathrm{L}}^{2}\right]
$$

In the pharmaceutical industry, the compressibility of pharmaceutical tablet formulations is an important factor which determines the required applied force on the composition of powder mixture to turn it into a structurally stable porous tablet. It greatly affects a range of tablet properties such as disintegration, dissolution, structural integrity, bioavailability and absorption as well as the mechanical properties, such as hardness and friability. The compressibility is defined as a mechanical property, which describes the relationship between the resulting compact density or strength (hardness / friability) and the compaction pressure [24].

We propose an "optical compressibility" parameter to estimate the mechanical compressibility of an excipient or complex formulation based on a simple analysis of the transmitted terahertz pulse. This "optical compressibility" is defined by using Eq. (1) as an optical state equation in analogy to the equation of state of a medium in thermodynamics. For the sake of clarity, we first consider the simple thermodynamic equation of state of an ideal gas, which is defined with the aid of the pressure $(p)$, volume $(V)$, absolute temperature $(T)$, the number of gas molecules (v) and the gas constant $(R)$ as $p V=v R T$. The optical state equation, namely Eq. (1), resembles the mathematical form of the thermodynamic state equation of an ideal gas, but obviously has different variables. The compressibility $\beta$, of an ideal gas is defined using the concept of a partial derivative as follows:

$$
\beta=-\frac{1}{V}\left(\frac{\partial V}{\partial p}\right)_{T}=\frac{1}{p}
$$

The unit of this compressibility is $\mathrm{Pa}^{-1}$. A definition similar to Eq. (6) can be exploited also for the compressibility of liquids and solids in the field of thermodynamics, but usually the state equation is more complicated than that of an ideal gas. The interpretation of Eq. (6) states that 
the higher the pressure, the lower is the value of the compressibility. In an analogous manner to Eq. (6), we define with the aid of Eq. (1) the optical compressibility parameter as

$$
\beta_{T H z}=-\frac{1}{H}\left(\frac{\partial H}{\partial n_{\mathrm{eff}}}\right)_{\Delta t}=\frac{1}{n_{\mathrm{eff}}-1}
$$

The dimensionless optical compressibility defined in this way shows inverse dependence of the compressibility on the effective refractive index, which in turn is linearly correlated to the density/porosity of the tablet. The interpretation of Eq. (7) is that the denser the medium (i.e. higher compaction pressure) the higher the effective refractive index, since the density of a medium is correlating with the refractive index of the medium, and, hence, the lower the optical compressibility parameter.

Next, we wish to have a more detailed picture regarding the behaviour of the optical compressibility parameter defined in Eq. (7). For this purpose, we consider an estimate for the explicit dependence of the optical compressibility on porosity, intrinsic refractive index of the excipient and the API, and also API mass fraction. An expression for the linear two-variable $\left(f_{\text {air }}, x\right)$ approximate effective refractive index of the tablet training sets of this study was given in [25] as follows:

$$
n_{\mathrm{eff}}=n_{\mathrm{MCC}}-\left(n_{\mathrm{MCC}}-1\right) f_{\mathrm{air}}-\left(n_{\mathrm{MCC}}-n_{\mathrm{API}}\right) x,
$$

where $n_{\mathrm{MCC}}$ and $n_{\mathrm{API}}$ are the intrinsic refractive indices of MCC and Indomethacin, $f_{\text {air }}$ is the porosity of the tablet, and $x$ is the dimensionless mass fraction (different from $f_{\mathrm{API}}$ ) of the API. By substituting Eq. (8) into Eq. (7), the optical compressibility can be re-expressed as,

$$
\beta_{\mathrm{THz}}=\frac{1}{\left(n_{\mathrm{MCC}}-1\right)\left(1-f_{\mathrm{air}}\right)-\left(n_{\mathrm{MCC}}-n_{\mathrm{API}}\right) x}
$$

Since $n_{\mathrm{MCC}}$ and $n_{\mathrm{API}}$ are constants, it is evident from Eq. (9) that $\beta_{\mathrm{THz}}$ is inversely dependent (hyperbolic dependence) on the porosity $f_{\text {air }}$ or mass fraction $x$ only when one of them is constant. In a general case, both porosity and the dimensionless mass fraction are considered to vary.

The optical compressibility $\beta_{\mathrm{THz}}$ depends on $S$ via Eq. (5). If we compare Eqs. (6) and (7), the message is pretty much similar. The thermodynamic compressibility $\beta$ becomes less as the pressure increases. In the case of increasing compression pressure in the tableting process the porosity of the tablet is decreasing and the effective refractive index is increasing, thus resulting in the decrease of $\beta_{\mathrm{THz}}$. The optical compressibility parameter $\beta_{\mathrm{THz}}$ and its connection to the $S$ structure parameter was studied for the training set of pharmaceutical tablets, and the results obtained will be shown below.

\section{Materials and methods}

Two sets of round flat-faced pharmaceutical tablets were compressed from the defined mixtures of pharmaceutical excipient MCC (Avicel PH101, FMC BioPolymer, Philadelphia, 
USA) and API Indomethacin (Hangzhou Dayangchem Co. Ltd., Hangzhou, China). The widely used MCC is a typical hydrophilic excipient [21], the nominal particle size and true density of the particulate Avicel PH101 are $50 \mu \mathrm{m}$ and $1.55 \mathrm{~g} \mathrm{~cm}^{-3}$, respectively. The true density of the crystalline gamma polymorph of Indomethacin used in this study is $1.37 \mathrm{~g} \mathrm{~cm}^{-3}$. Two training sets of flat-faced tablets of constant diameter $13 \mathrm{~mm}$ were compacted using a compaction simulator (PuuMan, Kuopio, Finland). More details on the sample preparation of the tablets were described previously [26-28]. In Tables 1 and 2, various properties of the training tablet sets are presented. In tablet Set 1, porosity and API mass fractions were kept constant at ca. 36 $\%$ and $10 \mathrm{wt} \%$, respectively, whereas both were varied for the case of the tablet Set 2 . For both tablet sets, five tablets were compressed for each sample number and the given values in Tables 1 and 2 are the average values of 5 tablets belonging to a given tablet number. For each sample, statistical errors in the calculations made for the nominal porosities are as follows: diameter \pm $0.008 \mathrm{~mm}$, height $\pm 0.005 \mathrm{~mm}$ (standard deviation of the sample mean), weight $\pm 0.01 \mathrm{mg}$ (readability and sensitivity of the scale), effective refractive index \pm 0.002 (by assuming a temporal resolution of $0.02 \mathrm{ps}$ ) and porosity $\pm 0.2 \%$ (calculated using the error propagation law).

Here we report on two case studies related to the lumped structural $S$ parameter and the optical compressibility as follows: Case 1; fixed porosity and fixed API mass fraction but variable height, and Case 2; varied porosity, API mass fraction and height. To calculate the $S$ parameter, presented in Tables 1 and 2, we have to utilise Eqs. (1) - (5). In order to solve Eqs. (3) and (4), we have to know the zero porosity refractive indices of $n_{\mathrm{MCC}}$ and $n_{\mathrm{API}}$, namely, $n_{\mathrm{MCC}}=1.86$ and $n_{\mathrm{API}}=1.73$. The latter value of zero porosity estimate of the refractive index of $n_{\mathrm{API}}$ is a better estimate than the one given in [25]. The zero porosity estimates of the refractive index of MCC and API were obtained by the linear extrapolation technique method as used in $[20,25,28]$. The density of the samples was calculated from the average dimensions and the average measured weight of the tablet. The tablet porosity was calculated by forming a ratio between the tablet density and the true density of MCC and Indomethacin, and the $S$ parameter was calculated by using the equations given in the theory section. In Table 1, we have numbered the samples according to the order of the increase of the tablet height, not the order of increase of the effective refractive index.

(1)

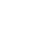


Table 1: Data of tablet Set 1. The mean values of the diameter $d$, height $H$, weight $W$, porosity $f_{\text {air, }}$, effective refractive index $n_{\text {eff, }}$ API mass fraction $w \mathrm{t} \%(x)$ and calculated $S$ parameter for four samples are shown. Since the porosity for all of the tablet samples is known, it is possible to calculate the volume fractions of MCC and API, as was discussed in [20].

\begin{tabular}{llllllll}
\hline $\begin{array}{l}\text { Sample } \\
\text { number } \\
(\text { Set } 1)\end{array}$ & $\begin{array}{l}d \\
(\mathrm{~mm})\end{array}$ & $\begin{array}{l}H \\
(\mathrm{~mm})\end{array}$ & $\begin{array}{l}W \\
(\mathrm{mg})\end{array}$ & $\begin{array}{l}f_{\text {air }} \\
(\%)\end{array}$ & $n_{\text {eff }}$ & $\begin{array}{l}x \\
(\mathrm{wt} \%)\end{array}$ & $S$ \\
\hline 1 & 13.097 & 2.742 & 361.47 & 36 & 1.529 & 10 & 0.220 \\
2 & 13.078 & 3.333 & 438.73 & 36 & 1.533 & 10 & 0.206 \\
3 & 13.066 & 3.626 & 476.45 & 36 & 1.537 & 10 & 0.194 \\
4 & 13.062 & 3.927 & 514.70 & 36 & 1.535 & 10 & 0.198 \\
\hline
\end{tabular}

Table 2: Data of tablet Set 2. The values of the diameter $d$, height $H$, weight $W$, porosity $f_{\text {air }}$,

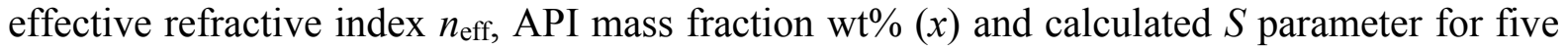
pharmaceutical tablets are shown.

\begin{tabular}{llllllll}
\hline $\begin{array}{l}\text { Sample } \\
\text { number } \\
(\text { Set } 2)\end{array}$ & $\begin{array}{l}d \\
(\mathrm{~mm})\end{array}$ & $\begin{array}{l}H \\
(\mathrm{~mm})\end{array}$ & $\begin{array}{l}W \\
(\mathrm{mg})\end{array}$ & $\begin{array}{l}f_{\text {air }} \\
(\%)\end{array}$ & $n_{\text {eff }}$ & $\begin{array}{l}x \\
(\mathrm{wt} \%)\end{array}$ & $S$ \\
\hline 1 & 13.076 & 3.955 & 404.02 & 50 & 1.405 & 11.00 & 0.271 \\
2 & 13.075 & 3.642 & 403.64 & 46 & 1.441 & 10.50 & 0.253 \\
3 & 13.094 & 3.273 & 405.67 & 40 & 1.498 & 10.00 & 0.219 \\
4 & 13.093 & 2.971 & 404.23 & 34 & 1.551 & 9.50 & 0.201 \\
5 & 13.081 & 2.734 & 406.20 & 28 & 1.602 & 9.00 & 0.194 \\
\hline
\end{tabular}

Mercury intrusion measurements were conducted using an Autopore V mercury porosimeter (Micromeritics Instrument Corporation, Norcross, GA, U.S.A.). The maximum applied pressure of mercury is $414 \mathrm{MPa}$, equivalent to a Laplace throat diameter of $4 \mathrm{~nm}$. The equilibration time at each of the increasing applied pressures of mercury is set to $20 \mathrm{~s}$. The tablets are measured as supplied.

240 By observing the behaviour under intrusion and extrusion at the highest pressures it is possible 241 to ascertain whether the sample displays the typical pore retention hysteresis or whether 242 mercury is extruded initially at equal volume to that during intrusion as a function of pressure. 243 If the latter occurs, then it is possible to conclude that the skeletal material is being elastically 
compressed, and the gradient of the elastic response to pressure provides a measure of the elastic bulk modulus of the skeletal material, i.e. the material bulk modulus of the pore wall when compressed equally from all directions. If the extrusion, however, exceeds the intrusion then the skeletal material is partially undergoing strong plastic deformation. The plastic deformation, however, is generally impossible to quantify as it is convoluted with the usual mercury retention hysteresis due to necking and filament snapping, and ink bottle behaviour. Thus, correcting for the elastic behaviour in the data can be included in the overall data correction during the mercury intrusion comprising the more commonly known effects of compression of mercury and expansion of the penetrometer [1]. This is performed conveniently using the software Pore-Comp (a software program developed by and obtainable from the Environmental and Fluids Modelling Group, University of Plymouth, U.K.), in which the following equation is applied:

$$
V_{\text {int }}=V_{\text {obs }}-\delta V_{\text {blank }}+\left[0.175\left(V_{\text {bulk }}^{1}\right) \log _{10}\left(1+\frac{P}{1820}\right)\right]-V_{\text {bulk }}^{1}\left(1-\Phi^{1}\right)\left(1-\exp \left[\frac{\left(P^{1}-P\right)}{M_{\text {ss }}}\right]\right)
$$

where $V_{\text {int }}$ is the volume of intrusion into the sample, $V_{\text {obs }}$ the intruded mercury volume reading, $\delta V_{\text {blank }}$ the change in the blank run volume reading, $V_{\text {bulk }}^{1}$ the sample bulk volume at atmospheric pressure, $P$ the applied pressure, $\Phi^{1}$ the porosity at atmospheric pressure, $P^{1}$ the atmospheric pressure and $M_{\mathrm{ss}}$ the bulk modulus of the solid sample [29].

\section{Results and discussion}

The values for the optical compressibility parameter, $\beta_{\mathrm{THz}}$, for the case of Set 1 are shown in Fig. 1 as a function of $S$. The porosity and API mass fraction were kept constant and only the height of the tablets was increased in this Set 1, which causes an increase in the volume of the tablet. The increase of the volume can be a probable reason for differences in arrangement of the particles in the direction of the THz pulse propagation, and hence different values of the lumped structural parameter $S$. From Table 1 it is evident that the refractive index of the tablets vary only slightly, whereas much stronger variations can be observed for their structural parameter $S$, sensitive to the series-parallel arrangement of constituents in the tablets.

Assuming that all the tablets of Set 1 have the same porosity and API wt $\%$, the conclusion can be drawn that a different share of series and parallel arrangement of the skeleton structure of the tablets contributes to slightly different values of the effective refractive index, and that this is manifested by a rather big change in the value of $S$. Therefore, $S$ has a descriptor role regarding also the compressibility of a tablet. Actually, the different heights of the nominally similar tablets of Set 1 generate essentially different shares of series and parallel structures and, hence, different values of $S$. In other words, the packing of API and MCC is different according to the different tablet heights, and thus, compression.

For the samples in the case of Set 2 we repeated the same analysis procedure as above. Note that in this case the samples follow a different numbering rule, to the extent that when the tablet height is decreasing (the volume of the tablet becomes smaller) the effective refractive index is increasing, as shown in Table 2. In Fig. 2 we plot the structural parameter as a function of the porosity for Set 2 . Here, dependence of the structural parameter $S$ on the porosity suggests 
a nonlinear relationship, which can be mathematically deduced from Eqs. (3) - (5). The range of variation of $S$ in this case of Set 2 is ca. $0.194-0.271$, which is much wider than for the case of Set 1, ca. $0.198-0.220$. In the case of Set 2, porosity and API are both subject to being varied. A wider range of porosity change suggests also a wider range of the magnitude of the structural parameter $S$.

The optical compressibility parameter $\beta_{\mathrm{THz}}$ as a function of $S$ for tablet Set 2 is shown in Fig. 3. The optical compressibility $\beta_{\mathrm{THz}}$ is increasing with increasing $S$. The optical compressibility range of Set 2 is ca $1.66-2.47$, which is wider than that of Set 1 ca. $1.86-1.89$. Since both the porosity and the API mass fraction have been changing in the situation of Set 2, it is necessarily more complex than in the case of Set 1.

Besides the correlation of $\beta_{\mathrm{THz}}$ with the parameter $S$ we also studied the explicit dependence of $\beta_{\mathrm{THz}}$ on $f_{\text {air }}$ and $x$ (shown in Figs. 4 and 5). Fig. 4 suggests a nonlinear, hyperbolic dependence of $\beta_{\mathrm{THz}}$ on $f_{\text {air. }}$ This is consistent with the estimate given in Eq. (9), namely a hyperbolic dependence of $\beta_{\mathrm{THz}}$ on $f_{\text {air. }}$. The data of Fig. 5 show apparently a weaker nonlinearity in respect to the dependence on $x$. However, if the mass fraction $x$ would have a wider scale of variation, hyperbolic dependence of $\beta_{\mathrm{THz}}$ on $x$ would also be expected.

Fig 6 shows the calculated optical compressibility parameter as a function of the measured mechanical parameter, namely skeletal bulk modulus. It is obvious that there is a correlation between the optical compressibility and the skeletal bulk modulus. The change of the optical compressibility is relatively strong as a function of the skeletal bulk modulus if we compare the samples 3-5 of this set 2, which present low porosity tablets with the lowest API loadings amongst the present samples.

The skeletal bulk modulus of the sample number 1 of Set 2 (Table 2) has the highest value and so the least compressible skeletal solid material of the five samples. This sample has the highest API loading, and so the ratio of API to compressible excipient is the highest. Sample number 5 of Set 2 (Table 2) in turn has the most compressible skeletal material corresponding with the lowest API loading. The API, therefore, has a high material bulk modulus, and in ratio with the more compressible excipient determines the observed compressibility of the tablet structure.

\section{Conclusions}

Compressibility of a pharmaceutical tablet is an important tablet property. The problem of measuring compressibility of a tablet is challenging because one needs to detect the change in volume of a tablet as a function of the compression pressure. This means, typically, that special measurement arrangements have to be realised under well-controlled laboratory conditions. Our idea outlined in this paper is to retrieve information on compressibility and, hence, mechanical properties of a tablet using a non-destructive method based on the THz pulse delay detection. In this article, we have introduced the concept of optical compressibility of pharmaceutical tablets.

The optical compressibility was studied for two training tablet sets. A theoretical model that gives explicit dependence of the optical compressibility of porosity and API mass fraction was given. The tablets of two differently compressed sets consisted of one excipient, MCC, and one 
API, Indomethacin. For the purpose of describing their compressibility, we derive the concept of optical compressibility based on the effective refractive index of a tablet. The difference between the conventional and optical compressibility is that in the latter case there is, in principle, no longer a need to evaluate physical compressibility by detection of any pressureinduced volume change of the tablet. However, there is a valuable subtlety arising from the change in packing structure as a function of unidirectional compression. This is seen in a change of the parallel to series coordination of the skeletal material as monitored by the lumped parameter structure factor $S$. Thus, it is possible to derive a compressibility using the optical approach, and that this optical compressibility is unique to the excipient-API formulation ratio in that the compression of the tablet leads to a change in effective refractive index together with a unique packing change. Thus, the combination of $n_{\text {eff }}$ and $S$ as a function of compressive force provides a quality control tool for both tablet compression and formulation consistency. The transmitted terahertz signal, therefore, gives volumetric and structural information on the tablet as it stands without using any external disturbance. In other words, the optical compressibility is an intrinsic property of each tablet and its formulation.

Relatively regular behaviour of the optical compressibility as a function of the structural parameter, $S$, porosity $f_{\text {air }}$ and API mass fraction $x$, was obtained for both tablet Sets 1 and 2 . Using the data of skeletal bulk modulus of Set 2 we found a correlation between the optical compressibility and bulk modulus. The bulk modulus relates only to the direct compressibility of the material itself making up the skeleton but not that of the skeleton structure.

The study of the structural parameter, as well as the optical compressibility provides a more comprehensive picture of the properties of a pharmaceutical tablet, and in principle can be used to understand better the mechanical properties such as strain, Young's modulus, Poisson's ratio etc. of a tablet.

Finally, we wish to remark that both the lumped structural parameter as well as the optical compressibility are suggested to be proportional to the surface roughness of a tablet [21]. It was demonstrated previously that the effective refractive index of tablets is proportional to the measured average surface roughness. This observation may open new ways to predict various properties of tablets by terahertz measurements in reflection setting, i.e. reflection of the $\mathrm{THz}$ pulse. Such a concept would be particularly beneficial when the API or excipient strongly absorbs $\mathrm{THz}$ radiation, rendering transmission measurements unfeasible.

\section{References}

[1]. M. E. Aulton, "Pharmaceutics the science of dosage from design", $2^{\text {nd }}$ Edn. ,Churchill Livingstone, Edinburgh, 2002.

[2]. H. Lieberman, L. Lachman, J. B. Schwartz, "Pharmaceutical Dosage Forms Tablets", Vol. 3, $2^{\text {nd }} E d n$. Marcel Dekker, New York, 1990.

[3]. F. P. Knudsen, "Dependence of Mechanical Strength of Brittle Polycrystalline Specimens on Porosity and Grain Size”, J. Am. Ceram. Soc., 42, 376 - 387, 1959. 
[4]. R. M. Spriggs, "Expression for effect of porosity on elastic modulus of polycrystalline refractory materials”, particulary aluminium oxide, J. Am. Ceram. Soc., 44, 628 - 629, 1961.

[5]. J. T. Fell, J. M. Newton, "Determination of tablet strength by the diametralcompression test”, J. Pharm. Sci., 59, 688 - 691, 1970.

[6]. G. Alderborn, Tablets and compaction. In: Aulton, M.E. (Eds), "Pharmaceutics the Science of Dosage Form Design, $2^{\text {nd }}$ Edn. Churchill Livingstone, Edinburgh, 2002.

[7]. J. A. Choren, S. M. Heinrich, M. B. Silver-Thorn, "Young's modulus and volume porosity relationships for additive manufacturing applications", J. Mat. Sci., 48, 5103 - 5112, 2013.

[8]. K. E. Peiponen, P. Bawuah, M. Chakraborty, M. Juuti, J. A. Zeitler and J. Ketolainen "Estimation of Young's modulus of pharmaceutical tablet obtained by terahertz timedelay measurement”, Int. J. Pharm, 2015.

[9]. D. Zhou, Y. Qiu, "Understanding Material Properties in Pharmaceutical Product Development and Manufacturing: Powder Flow and Mechanical Properties", J. Valid. Techn., 65 - 77, 2010.

[10]. C. K. Tye, C. (Calvin) Sun, G. E. Amidon, "Evaluation of the effects of tableting speed on the relationships between compaction pressure, tablet tensile strength, and tablet solid fraction”, J. Pharm. Sci., 94, 465 - 472, 2005.

[11]. A. Hart, "Effect of Particle Size on Detergent Powders Flowability and Tabletability", Chem. Eng. Process Techn., 6, 1- 4,2015.

[12]. R. W. Heckel, “Density-pressure relationship in powder compaction”, Trans. Metall. Soc. AIME, 221. 671 - 675. 1961.

[13]. H. Leuenberger, B. D. Rohera, "Fundamentals of Powder Compression. I. The Compactibility and Compressibility of Pharmaceutical Powders”, Pharm. Res., 3, 12 22, 1986.

[14]. R. Ishino, H. Yoshino, Y. Hirakawa, K. Noda, "Influence of tabletting speed on compactibility and compressibility of two direct compressible powders under high speed compression” Chem. Pharm. Bull., 38, 1987 - 1992, 1990.

[15]. S. Patel, A. M. Kaushal, A. K. Bansal, "Effect of Particle Size and Compression Force on Compaction Behavior and Derived Mathematical Parameters of Compressibility”, Pharm. Res., 24, 111 - 124, 2007.

[16]. D. H. Choi, N. A. Kim, K. R. Chu, Y. J. Jung, J-H. Yoon, S. H. Jeong, "Material Properties and Compressibility Using Heckel and Kawakita Equation with Commonly Used Pharmaceutical Excipients”, J. Pharm. Inv., 40, 237 - 244, 2010. 
400

401

402

403

404

405

406

407

408

409

410

411

412

413

414

415

416

417

418

419

420

421

422

423

424

425

426

427

428

429

430

431

432

[17]. K. S. Khomane, P. K. More, G. Raghavendra, A. K. Bansal "Molecular Understanding of the Compaction Behavior of Indomethacin Polymorphs”, Mol. Pharm., 10, 631-639, 2013.

[18]. I. M. Hamad, A. M. A. Salman, S. Elkarib, W. A. M. A. Salman, "The Effect of Particle Size on Compressibility of Cinnamon Granules”, J. Appl. Indust. Sci., 3, 150 - 153, 2015.

[19]. K. E. Peiponen, J. A. Zeitler, M. Kuwata-Gonokami (Eds.) Terahertz Spectroscopy and Imaging (Springer, Berlin, 2013).

[20]. P. Bawuah, M. Chakraborty, T. Ervasti, J. A. Zeitler, J. Ketolainen, P. A.C. Gane. K. -E Peiponen, "A structure parameter for porous pharmaceutical tablets obtained with the aid of Wiener bounds for effective permittivity and terahertz time-delay measurement”, Int. J. Pharm, 506, 87 - 92, 2016.

[21]. M. Chakraborty, P. Bawuah, Nicholas Tan, T. Ervasti, P. Pääkkönen, J. A. Zeitler, J. Ketolainen, K-E. Peiponen, "On the Correlation of Effective Terahertz Refractive Index and Average Surface Roughness of Pharmaceutical Tablets” J Infrared Milli Terahz Waves, DOI 10.1007/s10762-016-0262-0, 2016.

[22]. D. E. Aspnes, "Local-field effects and effective medium theory: A microscopic perspective”, Am. J. Phys., 50,704-709 ,1982.

[23]. O. Krischer, W. Kast, Die Wissenschaftlichen Grundlagen der Trocknungstechnik. (Springer, Berlin, 1978).

[24]. O. A. Adetunji, M. A. Odeniyi, O. A. Itiola, "Compression, Mechanical and Release Properties of Chloroquine Phosphate Tablets containing corn and Trifoliate Yam Starches as Binders”, Trop. J. Pharm. Res., 5 ,589 - 596, 2006.

[25]. P. Bawuah, N.Tan, S. N. A. Tweneboah, T. Ervasti, J. Ketolainen, J. A. Zeitler, K. E. Peiponen, " Terahertz study on porosity and mass fraction of active pharmaceutical ingredient of pharmaceutical tablets”, Eur. J. Pharm. Biopharm. 105, 122 - 133, 2016.

[26]. T. Ervasti, P. Silfsten, J. Ketolainen, K. E. Peiponen, "A study on the resolution of a Terahertz Spectrometer for the assessment of the porosity of pharmaceutical tablets", Appl. Spectrosc., 66, 319 - 323, 2012.

[27]. P. Bawuah, A. Pierotic Mendia, P. Silfsten, P. Pääkkönen, T. Ervasti, J. Ketolainen, J. A. Zeitler, K. E. Peiponen, "Detection of porosity of pharmaceutical compacts by terahertz radiation transmission and light reflection measurement techniques”, Int. J. Pharm., 465, 70 - 76, 2014.

[28]. P. Bawuah, P. Silfsten T. Ervasti, J. Ketolainen, J. A. Zeitler, K-E Peiponen, “Noncontact weight measurement of flat-faced pharmaceutical tablets using terahertz transmission pulse delay measurements”, Int. J. Pharm., 476, 16-22, 2014. 
433 [29]. P.A.C. Gane, J.P. Kettle, G.P. Matthews, C.J. Ridgway, Void Space Structure of

436

437

438

439 
Figure captions

Fig. 1: Optical compressibility $\beta_{\mathrm{THz}}$ as a function of structural parameter $S$ for Set 1 . Fig. 2: Structural parameter $\mathrm{S}$ as a function of porosity $f_{\text {air }}$ for Set 2 .

Fig. 3: Optical compressibility $\beta_{\mathrm{THz}}$ as a function of structural parameter $S$ for Set 2 Fig. 4: Optical compressibility $\beta_{\mathrm{THz}}$ as a function of porosity $f_{\text {air }}$ for Set 2.

Fig. 5: Optical compressibility $\beta_{\mathrm{THz}}$ as a function of mass fraction $x$ for Set 2.

Fig. 6: Optical compressibility $\beta_{\mathrm{THz}}$ as a function of Bulk modulus $M_{\mathrm{ss}}$ for Set 2 . 


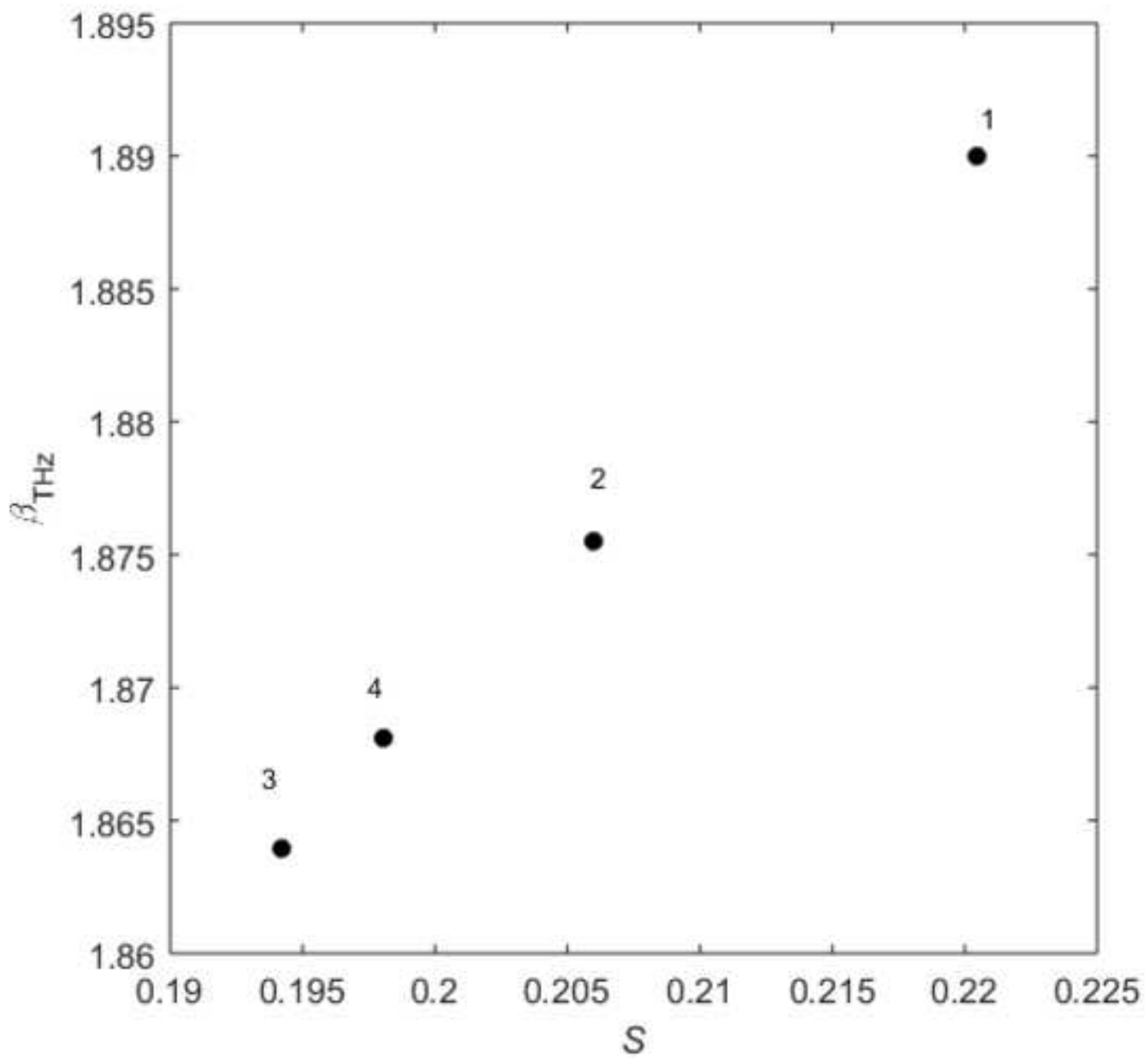




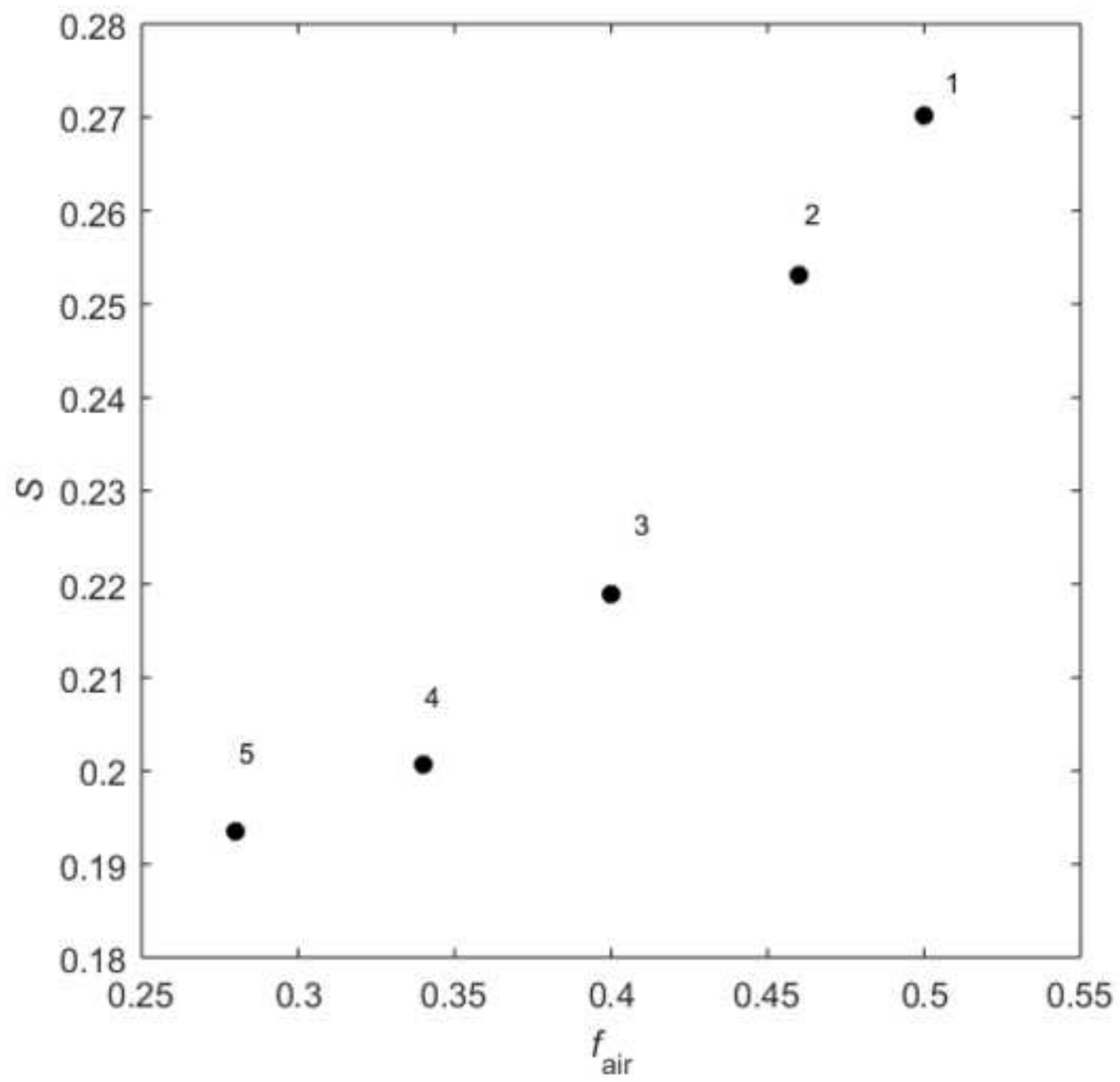


Figure(3)

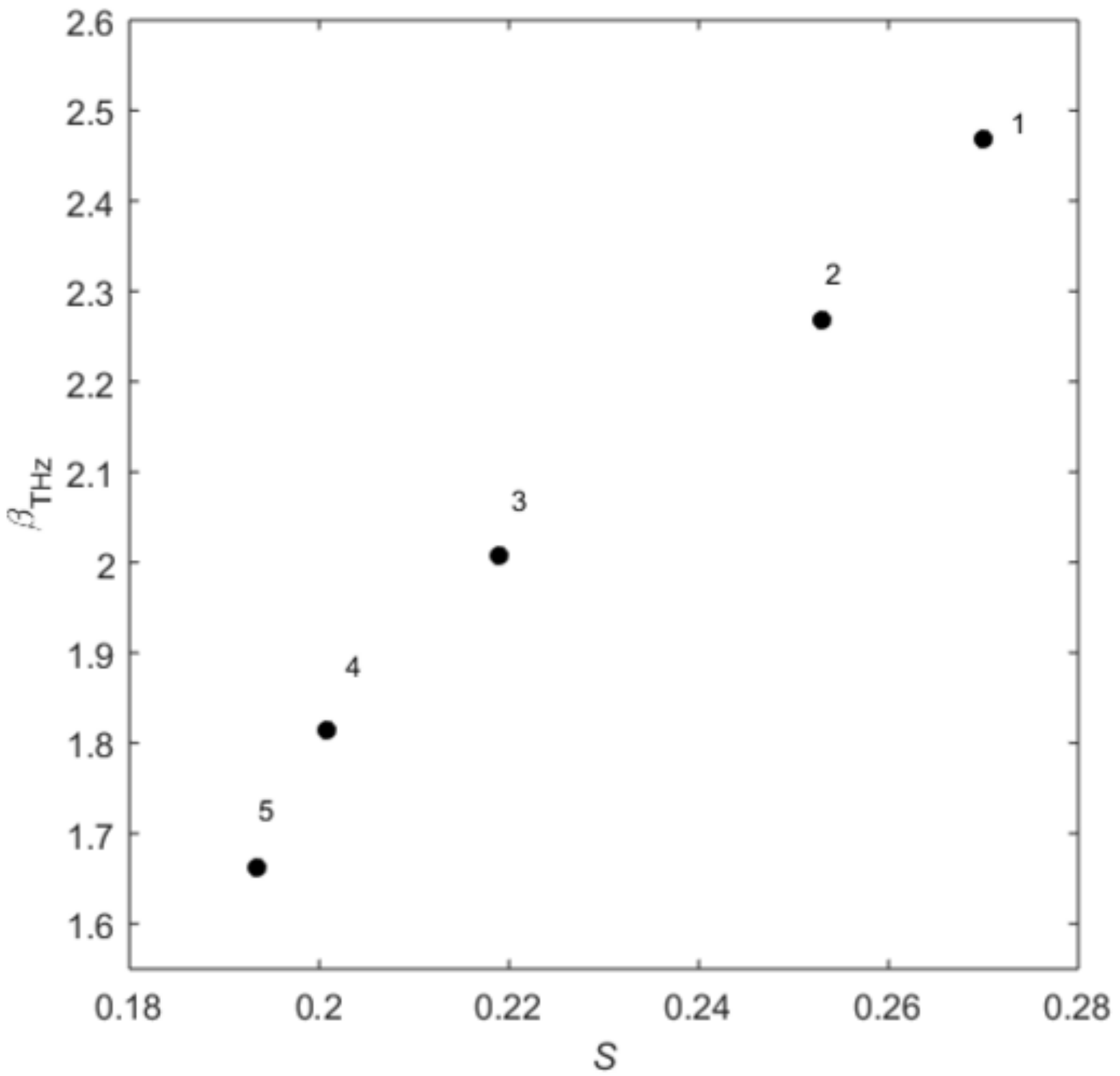




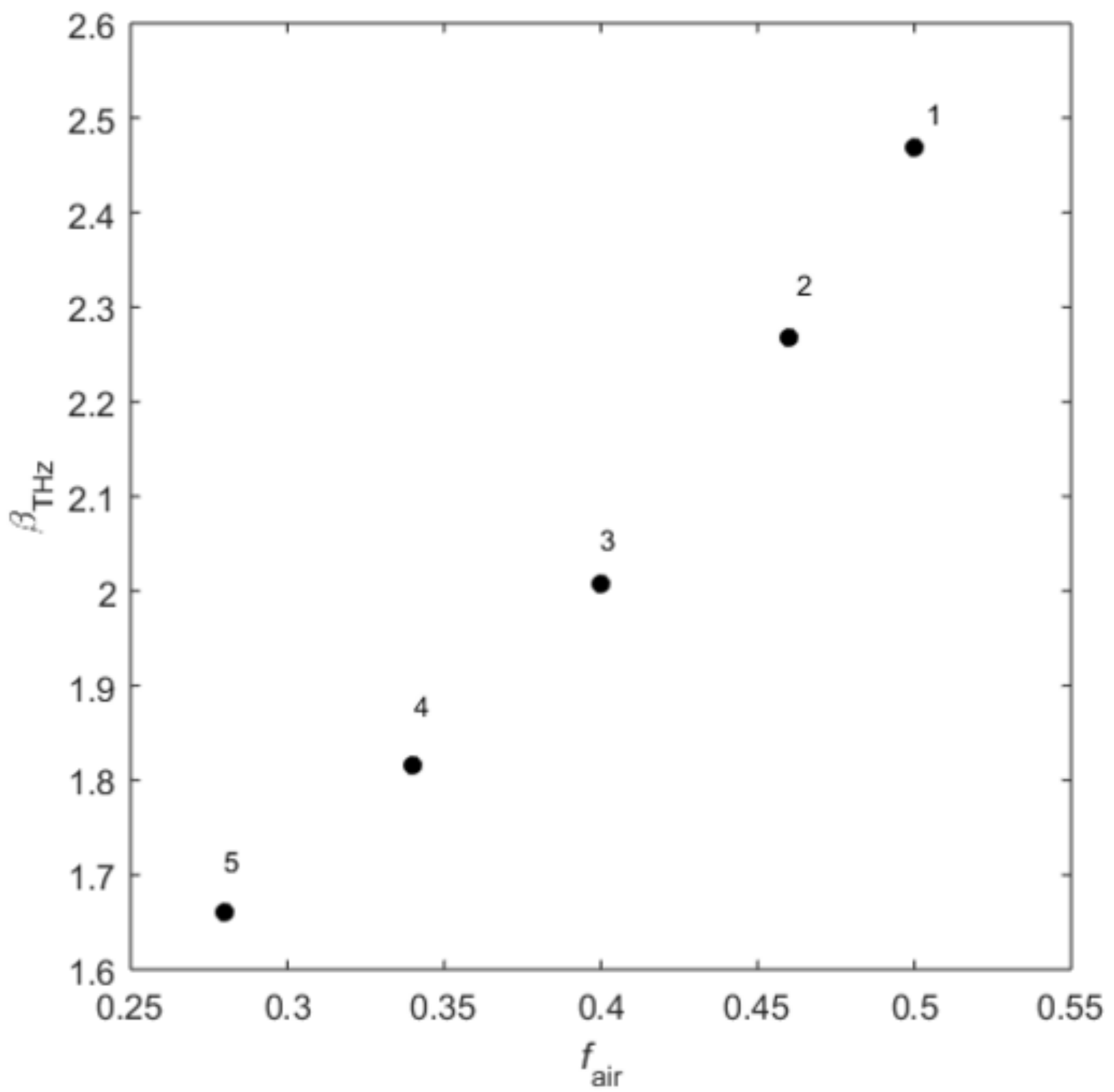




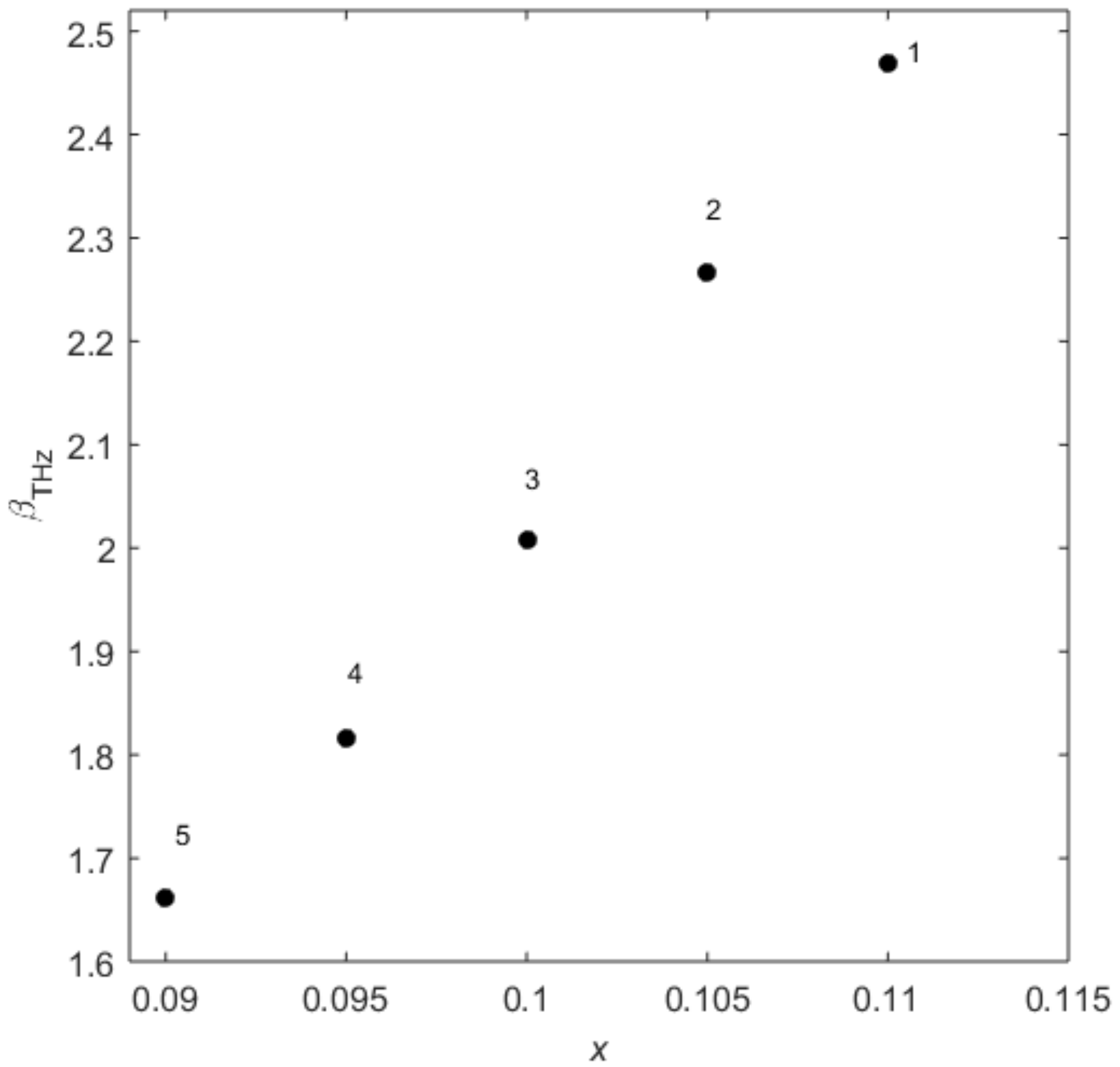




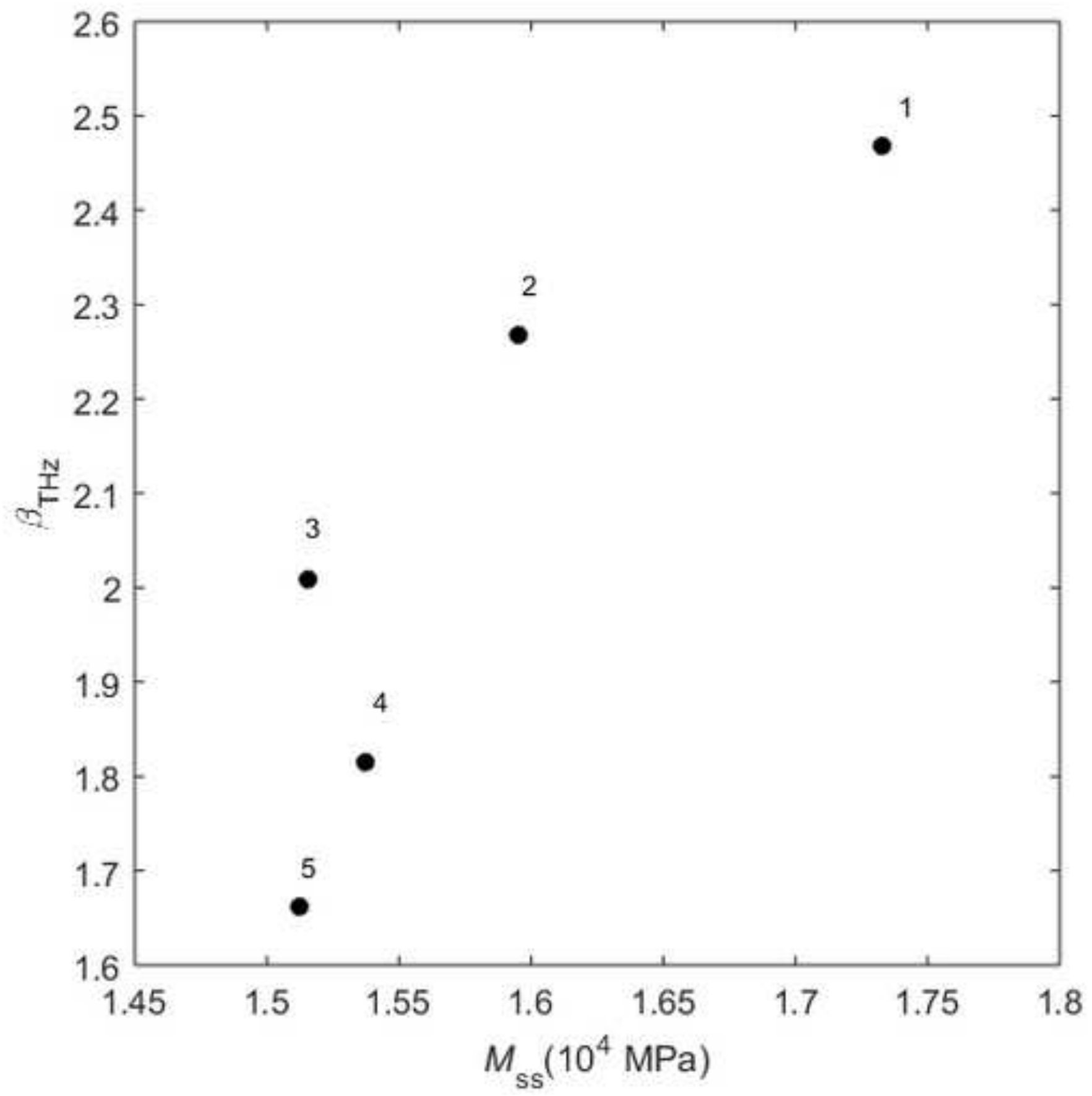

